\title{
Editorial
}

\author{
${ }^{*}$ Corresponding author \\ Zhonghua Sun, PhD, FSCCT \\ Professor \\ Department of Medical Radiation \\ Sciences, School of Science \\ Curtin University \\ GPO Box U1987, Perth \\ WA 6845, Australia \\ Tel. +61-8-9266 7509 \\ Fax: +61-8-9266 2377 \\ E-mail: z.sun@curtin.edu.au
}

\section{Volume 4 : Issue 1}

Article Ref. \#: 1000HROJ4e007

\section{Article History}

Received: January 21 $1^{\text {st }}, 2017$

Accepted: January $23^{\text {rd }}, 2017$

Published: January 23 ${ }^{\text {rd }}, 2017$

\section{Citation}

Sun Z. Quantitative assessment of coronary plaques by coronary $\mathrm{CT}$ angiography: High inter-reader and intra-reader agreement is achieved but inter-scanner variability should not be ignored. Heart Res Open J. 2017; 4(1): e1-e5. doi: 10.17140/HROJ-4-e007

\section{Copyright}

(C)2017 Sun Z. This is an open access article distributed under the Creative Commons Attribution 4.0 International License (CC BY 4.0), which permits unrestricted use, distribution, and reproduction in any medium, provided the original work is properly cited.

\section{Quantitative Assessment of Coronary Plaques by Coronary CT Angiography: High Inter-Reader and Intra-Reader Agreement is Achieved but Inter-Scanner Variability should not be Ignored}

\author{
Zhonghua Sun, PhD, FSCCT
}

Department of Medical Radiation Sciences, Curtin University, GPO Box U1987, Perth, WA 6845, Australia

\section{ABSTRACT}

This editorial discusses a recent paper published in the December issue of Radiology 2016 on the quantitative assessment of coronary plaques by coronary computed tomography (CT) angiography. Three main findings are discussed in the editorial: First, coronary CT angiography is an accurate imaging modality for analysis of plaque volume as well as monitoring volume change, with excellent inter- and intra-reader agreement with use of the state-of-the-art CT scanners. Second, the reliability of coronary CT angiography is noticed for low scan variability when plaque volume assessment is performed with the same vendor, but with high variability of nearly $30 \%$ when assessment is conducted with different vendors at baseline and follow-up scans. Finally, a large sample size is required to assess non-calcified plaques, in particular with use of different vendors.

KEY WORDS: Coronary artery disease; Coronary CT angiography; Coronary plaque; Volume measurement; Variability.

Coronary CT angiography (CCTA) is currently a widely used imaging modality for the diagnostic assessment of patients with suspected coronary artery disease (CAD) with high diagnostic accuracy reported in the literature. ${ }^{1-5}$ Rapid technological developments on cardiac CT imaging have occurred over the last decade which have led to the development and improvement of diagnostic spectrum of CCTA in the quantitative analysis of coronary plaques, in addition to the diagnostic value of coronary artery stenosis. These included characterization of plaque features and the corresponding clinical outcomes such as prediction of major adverse cardiac events, in addition to the routine assessment of coronary lumen stenosis. ${ }^{6-9}$

Detection of plaque components, in particular, differentiation of non-calcified (vulnerable) from calcified (stable) plaques is more significant than detection of lumen stenosis because close association has been reported between plaque composition and myocardial ischemia and development of adverse cardiac events. ${ }^{10-13}$ Therefore, the current research direction of CCTA has primarily focused on the quantitative assessment of plaque features instead of coronary lumen analysis because the degree of lumen stenosis is not always associated with myocardial ischemic changes..$^{14,15}$

Intravascular ultrasound (IVUS) and optical coherence tomography (OCT) are two imaging modalities which allow excellent plaque characterization by providing intravascular views of plaque components, however, both are invasive, thus, they are not commonly performed in clinical practice. As a less invasive modality with widespread use in daily practice, CCTA is able to provide quantitative assessment of plaque morphology and components which add 
incremental prognostic value in the diagnosis of CAD. ${ }^{16,17}$ There is a growing evidence to show that CCTA has good correlation with IVUS in the quantitative plaque analysis. ${ }^{18-22}$ However, limited research is conducted with regard to the inter/intra-reader and interscanner agreement or variability of CCTA in the quantitative assessment of coronary plaques. This has been addressed in a recently published study which is discussed in the following sections.

In the 2016 December issue of the Radiology, Symons et al ${ }^{23}$ investigated the variability of CCTA for quantifying plaque volume based on different latest multislice CT scanners. Authors prospectively enrolled 40 asymptomatic patients over 55 years old with history of hyperlipidemia who received medical treatment. Coronary artery calcium (CAC) scoring and CCTA were performed at baseline using 320-slice CT Toshiba Aquilion One scanner. Repeat CAC scoring and CCTA were performed with two different CT scanners within 30 days of the baseline scan, with 20 patients undergoing the repeat examinations using the same Aquilion One scanner, while another 20 patients using the Somatom Force 2x 192-slice dual-source scanner. Total plaque volume on a segment level and focal coronary lesion volume were measured and evaluated by two readers at baseline and follow-up scans. There was no significant difference in patient's demographics between the two groups undergoing different scans. High inter-reader and intrareader agreement (ICC-intraclass correlation coefficients $>0.99$ ) was noticed in the assessment of total, non-calcified and calcified plaques by CCTA for Toshiba and Siemens scanners. Scan-rescan variation was revealed when the follow-up CCTA was performed on a different type of CT scanner. The variability of CCTA was $18.4 \%$ and $16 \%$ for measurement of non-calcified plaque volume for all coronary arteries and for the most significant lesion, respectively when the baseline and follow-up CT scans were used with the same Toshiba CT. In contrast, the corresponding variability of CCTA was $29.9 \%$ and $26.4 \%$ when baseline scan was done on Toshiba CT and follow-up examination on Siemens CT, indicating high variability when follow-up CCTA was performed on a different type of scanner. The scan-rescan variation of CAC scoring was low with ICC $>0.99$ for both groups. Because of scanrescan variability in coronary plaque volume measurement, sample sizes were calculated for non-calcified plaque volume by CCTA. When a different vendor was used for the follow-up of plaque volume change, the estimated patients would be 587 and 753 for lesion-based and vessel-based analysis of non-calcified plaque, respectively.

There are three observations from Symons' study that bear discussions. First, the authors have demonstrated the accuracy and reliability of CCTA in the quantitative assessment of plaque volume. This finding is consistent with the current literature which supports the increasing use of CCTA for coronary plaque imaging analysis. Of different plaque features, low-attenuation area or non-calcified plaque represents one of the reliable indicators to determine plaque vulnerability and predict major adverse cardiac events. This has been confirmed by a number of studies showing good correlation between CCTA and IVUS in the differentiation of plaque composition. ${ }^{24-28}$ Main findings of these studies concluded that non-calcified or low-attenuating plaques were more often seen in patients associated with acute coronary syndrome or development of major adverse cardiac events when compared to those with stable angina pectoris. Therefore, assessment of non-calcified plaque in terms of total plaque volume has significant clinical value. The high inter-and intra-reader agreement as shown in Symons' study has further verified the diagnostic value of CCTA in this aspect.

The second comment is related to the variability of multislice CT scanner with regard to the plaque volume measurement. The two CT scanners used in this study included Toshiba 320-and Siemens Force scanners. The 320-slice scanner enables longitudinal coverage of $16 \mathrm{~cm}$, although the temporal resolution $(137.5 \mathrm{~ms})$ is not as good as that of dual-source CT scanners. ${ }^{4,29}$ A number of studies have demonstrated the high diagnostic value of 320 -slice CCTA in coronary artery disease, ${ }^{30-34}$ while Siemens Force is a $3^{\text {rd }}$ generation dual-source CT which is recently introduced into clinical practice with extended z-axis coverage and improved temporal resolution $(125 \mathrm{~ms})$. Recent studies have shown the improved diagnostic performance and lower radiation dose of $3^{\text {rd }}$ generation dual-source CT when compared to early generations of dual-source CT scanners. ${ }^{35,36}$ These two types of CT scans represent the latest technological developments in cardiac CT imaging and will continue to be used in clinical practice. Thus, findings from Symons study have significant clinical impact because patients with CAD after medical treatment will be followed up by CCTA using the latest CT scanners and these two vendors are the most commonly used in current practice. From a clinical perspective, Symons and colleagues in their study offered an insight into the scan-rescan variability when CCTA is performed with different vendors.

The third comment is related to the sample size calculation. Authors estimated the required sample size in order to detect $5 \%$ plaque volume changes by CCTA according to lesion-based and vessel-based analysis of non-calcified plaque when different vendors are involved in follow-up examinations. It is well known that the sample size plays an important role in study design and research findings. Most of the current studies are limited by small sample size or single center experience, which is frequently mentioned in the study limitations. The sample size estimation proposed by Symons and colleagues provides guidance for other researchers to develop similar studies so that robust conclusion can be drawn.

In summary, Symons and colleagues presented ${ }^{23}$ a very interesting study with further confirmation of high diagnostic accuracy of CCTA in the quantitative assessment of plaque volume with high inter- and intra-reader agreement. This study suggests 
that follow-up CCTA should be performed with the same vendor for diagnostic assessment of non-calcified plaque volume to avoid scan-rescan variability. A large sample size is required to detect plaque volume changes by CCTA when different vendors are involved at different periods of CT scans. Despite promising results presented in this study, further research, in particular, a longitudinal study with long-term follow-up of treatment outcomes is necessary to verify the findings.

\section{REFERENCES}

1. Budoff MJ, Dowe D, Jollis JG, et al. Diagnostic performance of 64-multidetector row coronary computed tomographic angiography for evaluation of coronary artery stenosis in individuals without known coronary artery disease: Results from the prospective multicenter ACCURACY (Assessment by Coronary Computed Tomographic Angiography of Individuals Undergoing Invasive Coronary Angiography) trial. J Am Coll Cardiol. 2008; 52(21): 1724-1732. doi: 10.1016/j.jacc.2008.07.031

2. Miller JM, Rochitte CE, Dewey M, et al. Diagnostic performance of coronary angiography by 64-row CT. $N$ Engl J Med. 2008; 359(22): 2324-2336. doi: 10.1056/NEJMoa0806576

3. Meijboom WB, Meijs MF, Schuijf JD, et al. Diagnostic accuracy of 64-slice computed tomography coronary angiography: A prospective, multicenter, multivendor study. J Am Coll Cardiol. 2008; 52(25): 2135-2144. doi: 10.1016/j.jacc.2008.08.058

4. Sun Z, Choo GH, Ng KH. Coronary CT angiography: Current status and continuing challenges. Br J Radiol. 2012; 85(1013): 495-510. doi: 10.1259/bjr/15296170

5. Xu L, Yang L, Fan Z, Yu W, Lv B, Zhang Z. Diagnostic performance of 320-detector CT coronary angiography in patients atrial fibrillation. Eur Radiol. 2011; 21(5): 936-943. doi: 10.1007/s00330-010-1987-0

6. Flohr TG, De Cecco CN, Schmidt B, Wang R, Schoepf UJ, Meinel FG. Computed tomographic assessment of coronary artery disease: state-of-the-art imaging techniques. Radiol Clin N Am. 2015; 53(2): 271-285. doi: 10.1016/j.rcl.2014.11.011

7. Saremi F, Achenbach S. Coronary plaque characterization using CT. AJR Am J Roentegenol. 2015; 204(3): W249-W260. doi: 10.2214/AJR.14.13760

8. Henzler T, Porubsky S, Kayed H, et al. Attenuation-based characterization of coronary atheroscleroticplaque: Comparison of dual source anddual energy CT with single-source CT and histopathology. Eur J Radiol. 2011; 80(1): 54-59. doi: 10.1016/j. ejrad.2010.07.024

9. Marwan M, Taher MA, El Meniawy K, et al. Invivo CT detection of lipid-rich coronary artery atherosclerotic plaques using quantitative histogram analysis: A head to head comparison with IVUS. Atherosclerosis. 2011; 215(1): 110-115. doi: 10.1016/j. atherosclerosis.2010.12.006

10. Pundziute G, Schuijf JD, Jukema JW, et al. Head-to-head comparison of coronary plaque evaluation between multislices computed tomography and intravascular ultrasound radiofrequency data analysis. JACC Cardiovasc Interv. 2008; 1(2): 176-182. doi: $10.1016 /$ j.jcin.2008.01.007

11. Lin F, Shaw LJ, Berman DS, et al. Multidetector computed tomography coronary artery plaque predictors of stress-induced myocardial ischemia by SPECT. Atherosclerosis. 2008; 197(2): 700-709. doi: 10.1016/j.atherosclerosis.2007.07.002

12. Pundziute G, Schuijf JD, Jukema JW, et al. Prognostic value of multislice computed tomography coronary angiography in patients with known or suspected coronary artery disease. J Am Coll Cardiol. 2007; 49(1): 62-70. doi: 10.1016/j.jacc.2006.07.070

13. Van Werkhoven JM, Schuijf JD, Gaemperli O, et al. Prognostic value of multislice computed tomography and gated singlephoton emission computed tomography in patients with suspected coronary artery disease. J Am Coll Cardiol. 2009; 53(7): 623-632. doi: $10.1016 /$ j.jacc.2008.10.043

14. Sun Z, Al Moudi M, Cao Y. CT angiography in the diagnosis of cardiovascular disease: A transformation in cardiovascular CT practice. Quant Imaging Med Surg. 2014; 4(5): 376-396. doi: 10.3978/j.issn.2223-4292.2014.10.02

15. Jia H, Abtahian F, Aguirre AD, et al. In vivo diagnosis of plaque erosion and calcified nodule in patients with acute coronary 
syndrome by intravascular optical coherence tomography. J Am Coll Cardiol. 2013; 62(19): 1748-1758. doi: 10.1016/j. jacc.2013.05.071

16. Maurovich-Horvat P, Ferencik M, Voros S, Merkely B, Hoffmann U. Comprehensive plaque assessment by coronary CT angiography. Nat Rev Cardiol. 2014; 11(7): 390-402. doi: 10.1038/nrcardio.2014.60

17. Nadjiri J, Hausleiter J, Jahnichen C, et al. Incremental prognostic value of quantitative plaque assessment in coronary CT angiography during 5 years of follow-up. J Cardiovasc Comput Tomogr. 2016; 10(2): 97-104. doi: 10.1016/j.jcct.2016.01.007

18. Sun Z, Xu L. Coronary CT angiography in the quantitative assessment of coronary plaques. Biomed Res Int. $2014 ; 2014: 346380$. doi: $10.1155 / 2014 / 346380$

19. Pflederer M, Marwan T, Schepis T, et al. Characterization of culprit lesions in acute coronary syndromes using coronary dualsource CT angiography. Atherosclerosis. 2010; 211(2): 437-444. doi: 10.1016/j.atherosclerosis.2010.02.001

20. Nakazato R, Shalev A, Doh JH, et al. Quantification and characterisation of coronary artery plaque volume and adverse plaque features by coronary computed tomographic angiography: A direct comparison to intravascular ultrasound. Eur Radiol. 2013; 23(8): 2109-2117. doi: 10.1007/s00330-013-2822-1

21. Papadopoulou SL, Neefjes LA, Schaap M, et al. Detection and quantification of coronary atherosclerotic plaque by 64-slice multidetector CT: A systematic head-to-head comparison with intravascular ultrasound. Atherosclerosis. 2011; 219(1): 163-170. doi: 10.1016/j.atherosclerosis.2011.07.005

22. Voros S, Rinehart S, Qian Z, et al. Prospective validation of standardized, 3-dimensional, quantitative coronary computed tomographic plaque measurements using radiofrequency backscatter intravascular ultrasound as reference standard in intermediate coronary arterial lesions: Results from the ALTANTA(Assessment of Tissue Characteristics, Lesion Morphology, and Hemodynamics by Angiography with Fractional Flow Reserve, Intravascular Ultrasound and Virtual Histology, and Noninvasive Computed Tomography in Atherosclerotic Plaques) I Study. JACCC ardiovasc Imaging. 2011; 4(2): 198-205. doi: 10.1016/j.jcin.2010.10.008

23. Symons R, Morris JZ, Wu CO, et al. Coronary CT angiography: Variability of CT scanners and readers in measurement of plaque volume. Radiology. 2016; 281(3): 737-748. doi: 10.1148/radiol.2016161670

24. Gauss S, Achenbach S, Pflederer T, Schuhback A, Daniel WG, Marwan M. Assessment of coronary artery remodelling by dualsource CT: A head-to-head comparison with intravascular ultrasound. Heart. 2011; 97: 991-997. doi: 10.1136/hrt.2011.223024

25. Takaoka H, Ishibashi I, Uehara M, Rubin GD, Komuro I, Funabashi N. Comparison of image characteristics of plaques in culprit coronary arteries by 64 slice CT and intravascular ultrasound in acute coronary syndromes. Int J Cardiol. 2012; $160(2)$ : 119-126. doi: $10.1016 /$ j.ijcard.2011.04.014

26. Motoyama S, Kondo T, Sarai M, et al. Multislice computed tomographic characteristics of coronary lesions in acute coronary syndromes. J Am Coll Cardiol.2007; 50(4): 319-326.

27. Motoyama S, Sarai M, Harigaya H, et al. Computed tomographic angiography characteristics of atherosclerotic plaques subsequently resulting in acute coronary syndrome. J Am Coll Cardiol. 2009; 54(1): 49-57. doi: 10.1016/j.jacc.2009.02.068

28. Vazquez-Figueroa JG, Rinehart S, Qian Z, et al. Prospective validation that vulnerable plaque associated with major adverse outcomes have large plaque volume, less dense calcium, and more non-calcified plaque by quantitative, three-dimensional measurements using intravascular ultrasound with radiofrequency backscatter analysis. Results from the ATLANTA I Study. $J$ Cardiovasc Trans Res. 2013; 6(5): 762-771. doi: 10.1007/s12265-013-9473-0

29. Meng L, Cui L, Cheng Y, et al. Effect of heart rate and coronary calcification on the diagnostic accuracy of the dual-source CT coronary angiography in patients with suspected coronary artery disease. Korean J Radiol. 2009; 10(4): 347-354. doi: 10.3348/ kjr.2009.10.4.347

30. Sun Z, Lin C. Diagnostic value of 320-slice coronary CT angiography in coronary artery disease: A systematic review and meta-analysis. Curr Med Imaging Rev. 2014; 10(4): 272-280. Web site. http://www.ingentaconnect.com/content/ben/ 
cmir/2014/00000010/00000004/art00008. Accessed January 20, 2017.

31. Pelliccia F, Pasceri V, Evangelista A, et al. Diagnostic accuracy of 320-row computed tomography as compared with invasive coronary angiography in unselected, consecutive patients with suspected coronary artery disease. Int J Cardiovasc Imaging. 2013; 29(2): 443-452. doi: 10.1007/s10554-012-0095-4

32. Tomizawa N, Maeda E, Akahane M, Torigoe R, Kiryu S, Ohtomo K. Coronary CT angiography using the second-generation 320-detector row CT: Assessment of image qualityand radiation dose in various heart rates compared with the first-generation scanner. Int J Cardiovasc Imaging. 2013; 29(7): 1613-1618. doi: 10.1007/s10554-013-0238-2

33. Wong DTL, Soh SY, Ko BS, et al. Superior CTcoronary angiography image quality at lower radiation exposure with second generation 320-detector row CT in patients with elevated heart rate: A comparison with first generation 320-detector row CT. Cardiovasc Diagn Ther. 2014; 4(4): 299-306. doi: 10.3978/j.issn.2223-3652.2014.08.05

34. Ghekiere O, Nchimi A, Djekic J, et al. Coronary computed tomography angiography: patient-related factors determining image quality using a second-generation 320-slice CT scanner. Int J Cardiol. 2016; 221: 970-976. doi: 10.1016/j.ijcard.2016.07.141

35. Meinel FG, Canstein C, Schoepf UJ, et al. Imaging quality and radiation dose of low tube voltage $3^{\text {rd }}$-generation dual-source coronary CT angiography in obese patients: A phantom study. Eur Radiol. 2014; 24: 1643-1650. doi: 10.1007/s00330-014-3194-X

36. Mangold S, Wichmann JL, Schoepf UJ, et al. Diagnostic accuracy of coronary CT angiography using $3^{\text {rd }}$-generation dual-source CT and automatic tube voltage selection: Clinical application in a non-obese and obese patient population. Eur Radiol. 2016 ; 1-11. doi: $10.1007 / \mathrm{s} 00330-016-4601-2$ 